Rev Biomed 2006; 17:152-154.

\title{
Coccidios intestinales en habitantes del Barrio 6 de Noviembre, Ciudad Bolívar, Estado Bolívar, Venezuela.
}

Carta al Editor

Rosario Tutaya, Ytalia Blanco, María Sandoval, Franegy Alcala, María Aponte, Rodolfo Devera.

Departamento de Parasitología y Microbiología, Escuela de Ciencias de la Salud. UDO-Bolívar. Ciudad Bolívar, Estado Bolívar, Venezuela.

Los coccidios intestinales son protozoarios tisulares obligatorios que habitan en la mucosa intestinal. Pertenecen al phylum Apicomplexa por presentar un Complejo Apical o aparato de penetración celular. De los varios géneros de coccidios, son patógenos el hombre Cryptosporidium, Cyclospora, Isospora, Sarcocystis y Toxoplasma. Los tres primeros tienen hábitat intestinal y causan criptosporidiosis, ciclosporiosis e isosporiosis, respectivamente (1).

La característica única de las infecciones por todos los coccidios intestinales es la presencia de ooquistes como forma infectante en las heces y que son el resultado del ciclo de reproducción que ocurre en el intestino delgado del hospedero. Además, estos ooquistes también constituyen la base del diagnóstico (1).

Es conocido que estas infecciones son más frecuentes en pacientes inmunocomprometidos, especialmente infectados con el Virus de la Inmunodeficiencia Humana, y en otros grupos de riesgo (2-5), pero también se pueden observar en población inmunocompetente o aparentemente sana $(1,6)$. Sin embargo, son pocos los estudios a nivel mundial, Latinoamericano y nacional (Venezuela) donde se ha empleado población aparentemente sana para determinar la prevalencia de coccidiosis intestinales (6). El objetivo del presente estudio, fue determinar la prevalencia de coccidios intestinales en un grupo de personas aparentemente sanas, habitantes de una comunidad urbana de Ciudad Bolívar, estado Bolívar, Venezuela.

Para ello, en mayo de 2003, se realizó un estudio trasversal de prevalencia donde se incluyeron a $100(24,2 \%)$ de los 413 habitantes del barrio 6 de Noviembre ubicado al norte de Ciudad Bolívar, estado Bolívar, Venezuela Se trata de una comunidad con precarias condiciones sociosanitarias y saneamiento ambiental deficiente.

Las 100 personas fueron evaluadas a través de interrogatorio y estudio coproparasitiologico de una muestra fecal mediante examen directo y métodos de Kato, formol-éter y coloración

Solicitud de sobretiros: Rodolfo Devera. Departamento de Parasitología y Microbiología, Escuela de Ciencias de la Salud, Universidad de Oriente, Núcleo Bolívar. Av. José Méndez. Ciudad Bolívar, Estado Bolívar, Código Postal: 8001-A. Venezuela. 


\section{R Tutaya, Y Blanco, M Sandoval, F Alcala, M Aponte, R Devera.}

de Kinyoun (7). Con los resultados obtenidos se construyó una base de datos con auxilio del programa SPSS 8.0 para Windows.

La edad de los 100 habitantes evaluados osciló entre 2 meses y 63 años, con una media de 17,1 años \pm 16 . El $65 \%$ de los evaluados eran menores de 14 años. Con respecto al género, 43 habitantes (43\%) eran masculinos y 57 (57\%) eran femeninos. Se encontró una prevalencia general de parasitosis intestinal de 63\% (63/100), donde se evidenció el predominio de los protozoarios (93\%). El 52,4\% (33/63) de los infectados resultó monoparasitado. El 57\% de los afectados eran del sexo femenino (36/63), siendo los individuos entre 10 y 14 años los más parasitados con 78,9\% (17/19). En ninguno de estos casos las diferencias encontradas fueron estadísticamente significativas $(\mathrm{p}>0,05)$

Un total de 13 especies de enteroparásitos fueron diagnosticados, destacando entre los protozoarios Blastocystis hominis (40\%) y Entamoeba coli (17\%); mientras que entre los helmintos el más común fue Trichuris trichiura con 7 casos (7\%) (cuadro 1). La prevalencia de coccidios intestinales fue de $10 \%$, siendo diagnosticados 9 casos de Cryptosporidium parvum y uno de Cyclospora cayetanensis. No fueron diagnosticados ooquistes de Isospora belli.

De los 10 casos de cocccidiosis, siete se observaron en el grupo de 5-9 años, dos en el de 10-14 y el uno en el grupo de 25-29 años. El sexo femenino fue más afectado (90\%) que el masculino. Siete de los 10 estaban asociados a otros parásitos, siendo mas comunes los protozoarios, destacando B. hominis y E. coli con 27,3\% cada uno. $\mathrm{Al}$ relacionar la consistencia de las heces con la presencia de coccidios intestinales se observó que sólo en un caso (10\%) las heces eran diarreicas y estaba parasitado por C. parvum. Los otros casos tenían heces de consistencia pastosa o blanda.

En conclusión, se encontró una elevada prevalencia de parasitosis intestinales (62\%); además, se determinó una relativa alta prevalencia

\section{Revista Biomédica}

(10\%) de coccidiosis intestinal en habitantes del Barrio 6 de Noviembre de Ciudad Bolívar. El coccidio intestinal más prevalente fue $C$. parvum con $9 \%$, siendo los niños los más afectados y el sexo femenino igualmente el más parasitado. La mayoría de los casos de coccidiosis cursaban de forma asintomática y asociados a otros protozoarios intestinales. Estos hallazgos vienen a confirmar las observaciones de varios autores que sostienen que aunque en bajo porcentaje, las personas inmunocompetentes pueden ser infectadas por estos coccidios intestinales $(6,8)$.

Cuadro 1.

Prevalencia de parásitos intestinales en habitantes del Barrio 6 de Noviembre, Ciudad Bolívar, Edo. Bolívar, Venezuela.

$\begin{array}{lll}\text { Parásitos } & \text { No. \% }\end{array}$

Protozoarios

Blastocystis hominis

$40 \quad 40$

Entamoeba coli

$17 \quad 17$

Endolimax nana

$14 \quad 14$

Giardia lamblia

$12 \quad 12$

Cryptosporidium parvum*

9

Iodamoeba butschlii

2

Chilomastix mesnilii

2

Pentatrichomonas hominis

2

Entamoeba histolytica/E. dispar

1

Cyclospora cayetanensis*

$1 \quad 1$

Helmintos

Trichuris trichiura

7

7

Ascaris lumbricoides

$1 \quad 1$

Strongyloides stercoralis

1

* En coloración de Kinyoun 


\section{Coccidios intestinales en Ciudad Bolívar, Venezuela.}

Trabajo parcialmente financiado por el Consejo de Investigación de la Universidad de Oriente, Proyecto CI-2-0407-1130/03.

Palabras clave: parásitos intestinales, epidemiología, coccidios, Crytosporidium parvum.

\section{REFERENCIAS.}

1.- Goodgame RW. Understanding intestinal spore-forming protozoa: Cryptosporidia, microsporidia, Isospora and Cyclospora. Ann Intern Med 1996; 124:429-41.

2.- Pape JW, Verdier R, Boncy J, Johnson W. Cyclospora infection in adults infected with HIV. Clinical Manifestations, Treatment, and Profilaxis. Ann Inter Med 1994; 121:6547.

3.- Chacín-Bonilla L, Bonilla M, Soto-Torres L, RiosCandida Y, Enmanuels C, Parra AM, et al. Cryptosporidium parvum in children with diarrhea in Zulia State, Venezuela. Am J Trop Med Hyg 1997; 56:365-9.

4.- Oshiro E, Cavalheiros M, Brandao V, Almeida M, Morelli L. Prevalencia do Cryptosporidium parvum em criancas abaixo de 5 anos, residentes na zona urbana de Campo Grande, MS, Brasil, 1996. Rev Soc Bras Med Trop 2000; 33:277-80.

5.- Nuñez FA, González O, González I, Escobedo E, Cordoví R. Intestinal Coccidia in Cuban Pediatric patients with diarrea. Mem Inst Oswaldo Cruz 2003; 98:539-42.

6.- Chacín-Bonilla L, Mejía de Young M, Estévez J. Prevalence and pathogenic role of Cyclospora cayetanensis in a Venezuelan community. Am J Trop Med Hyg 2003; 68: 304-6.

7.- Botero D, Restrepo M. Parasitosis humanas. 3ra ed. Medellín: Corporación para Investigaciones Biológicas. Medellín; 2003. p. 457.

8.- Eberhard ML, Nace EK, Freeman AR, Streit TG, Da Silva A, Lammie PJ. Cyclospora cayetanensis infections in Haiti: A common occurrence in the absence of watery diarrhea. Am J Trop Med Hyg 1999; 60:584-6. 